\title{
Repurposing learning objects: a sustainable alternative?
}

\author{
Cathy Gunn*, Sheila Woodgate and Winnie O'Grady \\ The University of Auckland, New Zealand
}

\begin{abstract}
Recent experience shows that reusable learning objects, like the computer assisted learning programmes of the early 1990s, have so far failed to achieve expected levels of integration into educational practice. This is despite technical interoperability, cataloguing systems, high quality standards, targeted dissemination and professional development initiatives. Analysis of this problem suggests that conceptualization of the problem may be limiting the scope of solutions. This paper proposes a sustainable and participative approach to reuse that involves repurposing learning objects for different discipline areas.
\end{abstract}

For some time now there has been a growing awareness that even the most accessible resources have failed to be widely adopted by the educational community and as a result have also failed to fulfil their considerable educational potential. (Campbell, 2003, p. 35)

\section{Introduction}

This quote from the book Reusing Learning Objects: A Sustainable Approach to E-learning (Littlejohn, 2003) describes the current state of progress with integration of digital learning objects into academic practice. Uptake of these resources is happening at a slower than desirable pace, despite the unquestionable quality of design and production, high levels of investment in professional development and the rationally anticipated outcome of this investment. The concept of reusability is not specific to digital objects as the widespread acceptance of resources such as textbooks, videos and educational software shows. The challenge of finding a route to widespread acceptance and reuse of digital learning objects is the focus of much attention in the current higher education context. This paper presents an alternative perspective on the concept of reusability that has so far received relatively little consideration. It may, in fact, be more accurately described as repurposing. It is based on the perfectly reasonable assumption

*Corresponding author. Cathy Gunn, Centre for Professional Development, The University of Auckland, Private Bag 92019, Auckland, New Zealand. Email: ca.gunn@auckland.ac.nz 
that teachers need to develop new and contextually appropriate instructional strategies for a learner-centric design model that is commonly associated with e-learning and the use of digital learning objects. Success depends on a relationship-based learning model where advances in knowledge are recognized as the product of combined objective and subjective elements. In this case, the objective element is information about learning objects as a generic concept. The subjective aspect is each individual teacher's emergent conceptual model of how learning objects can be integrated into an existing repertoire of discipline-based knowledge and teaching skills. The perceived benefits of developing new teaching strategies have to justify the investment of time and resources for such initiatives to be undertaken. In the case of digital learning objects, the discourse of objectivism and commodification surrounding their development is proving counter-productive to even conceptual acceptance of reusability. Grand-scale predictions followed by spectacular underachievement of profit-driven e-learning organizations, together with the association of e-learning with the failed 'dot.com economy', is enough to convince many observers that the learning object economy is just another passing fad.

A recently published report, 'Thwarted innovation: what happened to e-learning and why?' (Zemsky \& Massy, 2004), represents the voice of reason in saying that, now the initial euphoria has worn off, the true potential of e-learning and digital learning objects can begin to be realized. Those with experience in the field know that the combination of technology and pedagogy offers powerful potential to enhance student learning. What seems to be missing at this stage of the innovation cycle is the key that will allow a critical mass of teachers to unlock that potential. This article in no way seeks to denigrate other initiatives designed to meet this challenge. Rather, it presents a complementary approach that could move learning object reuse on to a higher level of acceptance.

\section{A sustainable approach to reusability}

A sustainable approach to reusability involves teachers in a participative design process that results in a sense of ownership, acceptance and ability to realize the potential of technology in different contexts. Underlying assumptions differ considerably from the 'course design as assembly of autonomous components' approach that has so far failed to achieve anticipated economic and educational objectives (Priest \& Quaife-Ryan, 2004). This difference may be critical if the goal is to move beyond the use of learning objects as supplementary materials within the context of long-established teaching models.

It may be pertinent to note that the accepted wisdom of developers of new technology rarely prove capable of making reliable predictions about its eventual use, (see for example, Burbules \& Callister, 2000). It is only when the intended (and sometimes unanticipated) end users of technology add their requirements, experience and professional practice that mainstream integration is achieved. The original technology 'object' remains the designer's creation, but the different 'versions' and uses that invariably emerge are developed by end users. If this is indeed the case, then the exact 
specification defined by learning object designers may not be suitable for all end users and customization may be required. Continuing this line of argument, it may be the structure or the content of a learning object that ultimately proves to be the reusable aspect. The majority of promotional activities to date have focused on reuse of complete objects with a fixed combination of structure, content and technical elements. While the value of this approach is not disputed, critical issues of deep, conceptual understanding, a sense of ownership and wider issues of cultural assimilation remain unresolved. These issues alone can determine the success or failure of educational innovations, regardless of technical robustness, accessibility and quality of content. The case presented here is that fully supported opportunities for teachers to 'repurpose' object structures through a participative design process is the path most likely to lead to the elusive goal of reuse of digital learning objects by a critical mass of teachers. Repurposing is defined as a process where the original structure of a learning object is populated with content from a different source and/or subject area and used to develop new learning activities.

\section{Defining learning objects}

Many definitions of reusable learning objects exist. A commonly accepted one is:

Any digital resource capable of reciprocal action that can be reused to support learning.

This definition combines key elements identified in two published sources. Wiley (2000, p. 4) offers a description that is at once simple, flexible and comprehensive:

Any digital resource that can be reused to support learning.

Nichols (2002, p. 2) adds the critical dimensions of student input and system reciprocity.

Learning objects are simulations or learning activities that are responsive to student input, that is, the objects provide some level of reciprocity and encourage constructivist forms of learning.

The reference to reciprocal action is key to focusing on learning as opposed to teaching objects. While this is not a commonly drawn distinction, these authors agree that it is an important one.

Teaching objects are primarily presentation based, but may use multiple media; such objects are not interactive in a reciprocal sense (i.e. they do not meaningfully respond to decisions made by the student) and have a cognitive bent. (Nichols, 2002, p. 2)

Most focus to date has been on making content available (teaching objects) rather than preparing interactive exercises (learning objects). This highlights the difference between teacher-centred and student-centred activity and a common misconception about effective e-learning; that is, that presentation of content in digital format is the ultimate goal, rather than student learning through active engagement with responsive online systems that may (or may not) include multimedia features. Some definitions of learning objects appear to support this misconception, and part of the reason 
for low levels of uptake may stem from the perception that learning objects are simply content material converted to a different format (e.g. from print to multimedia). The difference this makes to student learning is unlikely to justify major investment or to visibly support early adopters' claims of an emergent educational paradigm. In these circumstances, even the initial stages of Hurley's (1992) model of acceptance of new technology in organizations, (illustrated in the following diagram) (i.e. perceived usefulness and early participative involvement) may not be reached, or followed by the later stages of trials, acceptance and use.

Another risk noted by Hurley is:

... many examples exist where the central concept of acceptance appears to be studiously ignored, while somewhat peripheral concepts of a highly disparate nature are explored with great thoroughness. (Hurley, 1992, p. 29)

In the case of learning objects, debates about granularity, the relative merits of different technical specifications and architectural models continue. As noted by Campbell (2003), these are critical conditions for the successful development of the learning object economy. However, they are not the key factors that integration of learning objects into teaching practice depends on. If they were, the current 'low acceptance dilemma' would not be faced because solutions would already be well developed. Since matters of definition and technical description are peripheral to the case presented, no further discussion is included. These issues are addressed elsewhere in more detail (see for example, Wiley, 2000).

\section{Why reusable objects are not reused}

Investigations of the 'slow adoption' phenomenon have appeared in the literature for over a decade, with many reasons proposed and potential remedies tested. The underlying technology was somewhat different in the scenarios described by Gardner \& Darby (1990), Darby (1992a \& b), Hammond et al. (1992), Hodas (1996) and Taylor (1998), but the nature of the challenge was the same-that the anticipated rate of integration of computer-supported learning had failed to materialize. Investment in production, dissemination and professional development did not succeed in removing the barriers to uptake. In the case of reusable learning objects, the literature describes a complex list of issues and the means through which the barriers they create are addressed, including:

- Interoperability and cataloguing standards (for example, Wilson, 2001).

- Quality control for content (for example, Taylor \& Richardson, 2001).

- Inclusion of pedagogical frameworks (for example, Boyle \& Cook, 2001).

- Targeted professional development for teachers (for example, Littlejohn, 2003).

Given the relative success of these initiatives and the paradox of variable impact of such targeted investment, the question that follows is whether some fundamental limitation is imposed by current conceptions of the problem and the scope of proposed solutions. 


\section{Conditions for acceptance of new technology}

Before focusing on the case of digital learning objects, acceptance of new technology in organizations is considered for two reasons. Firstly it situates the learning objects dilemma in the broader field of organizational change (Fullan, 2001). Secondly it identifies generic influences in the specific context. While the models of authors such as Zemsky and Massy (2004) describe cycles of technology adoption, they do not identify influential factors at various stages. For this purpose, a different model is useful.

Hurley (1992) presents a psychological model for the acceptance of new technology, and notes that introduction rather than acceptance of new technology provides the focus for the bulk of research studies. Contemporary organizational theory supports Hurley's argument that rationalist viewpoints do not reflect the reality of change, where political, cultural and personal factors are as influential as management initiatives and strategic plans. The model is a simple, sequential one, shown in Figure 1. Details of the stages matter less to this case than the sequence of events and cyclical nature of the process.

The process may be uniform during the first three stages as early adopters move through the conceptual, experimental and trial phases. The next three stages are known to be met with varied levels of enthusiasm by different stakeholders and may take considerably longer. The final phase is subject to financial, political and cultural pressures that may bear little or no direct relationship to the merit of the new technology itself, so the issues are complex. The case study presented in this paper does not address these final stage complexities, although exclusion does not imply insignificance. The aim is simply to present an alternative way for the potential of technology, in this case learning objects, to be realized through teacher involvement in design.

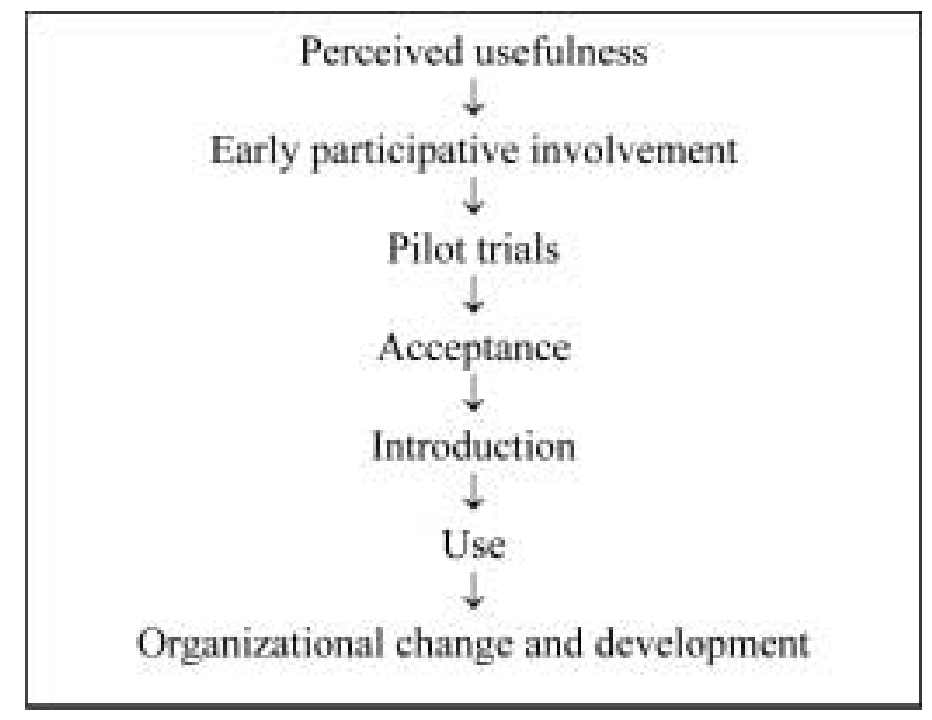

Figure 1. Process model of the introduction of new technology 


\section{Participation and change}

User participation in system design is beneficial for two reasons. System design is more likely to reflect specific user needs, and acceptance is easier when a sense of ownership evolves during the development phase. The 'not invented here syndrome' reported in the computer assisted learning literature of the 1990s describes many situations where pedagogically sound, technically robust and usable programmes were developed under government-funded initiatives such as TLTP (UK), and AUTC (Australia). The outcome was, in one important respect, similar to the current experience.

Despite coordinated efforts such as these to promote the effective use of computers in teaching, the uptake is not as high as many have hoped. (Hammond et al., 1992, p. 155)

The need for a conceptual shift is key to acceptance of learning objects that are developed by third parties. Downes' (2001) report 'Learning objects: resources for distance education worldwide' raises the point that, logically, the world does not need thousands of similar objects addressing basic educational concepts when one, or at most a dozen, would meet the needs of all learners. The economic argument for limited production is compelling, yet the reinvention process continues because this is the culturally familiar pattern of teacher behaviour repeating itself in the digital age. The perceived threat to teacher status that this poses is an obvious barrier to acceptance, and the limitations of the argument may be lost on inexperienced users or late adopters of technology-facilitated learning. Experienced users know that technology opens up creative opportunities to apply existing expertise to the challenges posed by the changing educational context.

So if logic and economics are not the determining factors, what does the reusability decision hinge on for individual academics? Further analysis of this question is possible without resorting to speculation. Using the example of resources that are frequently reused (i.e. textbooks, maps, software programs, graphics and videos), some common factors can be identified. One is that they are produced centrally and distributed widely by established publishers. Perhaps more importantly, the shared resources are used in parts rather than in entirety, and integrated into a wide variety of learning activities. While the learning objects themselves may be standard, the range of possible uses is far from uniform. It seems entirely feasible to suggest that digital objects could be used in similar ways; the key points being that teachers have sound conceptual models of what these reusable materials are, the extent of their performance and creative potential for combination and reuse for different purposes. Sometimes teachers are also authors, but in all cases the conceptual models are more or less complete. Most importantly, the objects neither depend on nor dictate the use of any particular teaching style or strategy, thus allowing teachers the freedom to implement their own.

\section{Design and participation}

The case now presented demonstrates how participation in the design phase of a repurposing process can provide a direct route through acceptance to organizational 
change and development. The conclusion is that one highly effective way to get learning object reuse into the repertoire of teachers is through the role of a collaborative developer. It may not be practical for every teacher to develop the technical skills to produce learning objects from scratch. However, working collaboratively with the structure of an existing object, populating it with familiar content and embedding it within self-defined learning activities results in many benefits:

- Production of a useful teaching resource.

- Knowledge of production processes.

- A sense of ownership.

- Confidence to use the learning object in a variety of appropriate situations.

- An increased level of knowledge of the synergies that result from bringing together technology, instructional design and teaching expertise.

Assuming the success of such a venture and a positive impact on student learning, it is reasonable to expect that progress through all stages of the acceptance model will result. If roles then shift, as they frequently do, the newly competent reusable learning object user may become a mentor for others, as initial evidence points to these skills being transferable.

\section{Skills for reuse}

Consider the case of a teacher using an existing learning object structure to develop resources to support learning within their own subject. Pedagogical content knowledge (Barnett \& Hodson, 2001) and instructional design skills already exist within the teacher's repertoire. These are necessary, although insufficient, conditions for effective use of learning objects. Knowledge of how technology can complement these skills must be added. In the process of repurposing an existing object structure, teachers develop conceptual understanding of the particular object as well as the generic concept of learning objects. If instead they choose to reuse an existing object, they might learn about its description in a cataloguing system, the content that is included and pedagogical contexts for use. It is unlikely, however, that they would be able to construct their own objects or to repopulate object structures as a result of this experience. Participative design puts the learning object structure and functionality in focus during the teacher's situated learning experience. Some evidence emerged from the case study, described in this paper, to suggest that this experience promoted a review of other aspects of learning activity design such as logical flow, being concise and tightly structured. The distinction between reuse and repurposing may appear subtle in some respects, while the outcomes differ significantly. One leads to acceptance and enhanced instructional design skill, the other to increased awareness of available resources and the ability to use a range of presentation techniques.

While participative design requires a period of intensive support from the developer, an education technologist or an instructional designer familiar with the object structure, the investment is justified by the outcomes. The relative neglect of a participative approach to reusability represents a significant remaining barrier to uptake. 
The potential success of this approach is illustrated through a collaborative venture between lecturers in chemistry and in accounting information systems (AIS).

\section{Case study: a problem in AIS and application to a reusable chemistry tutorial system}

This case study reflects a fairly typical scenario where an academic with some working knowledge of e-learning was motivated to seek a technology-based solution to a student learning problem. The challenge facing AIS learners was an apparent inability to translate narratives on design and interpretation of data flow diagrams into visual representations (i.e. diagram components) and appropriate structural combinations (i.e. complete functional diagrams). These skills cannot be rote learned for a number of reasons, and students have to reach a higher level of competence than what seemed to be possible in the limited contact time available. They need to understand the concept at a general level of abstraction rather than in specific detail, and experience shows experiential learning is effective. Devoting additional time to this basic topic would compromise overall progress, yet students clearly need more frequent engagement and timely constructive feedback than is provided in class. The teacher had the content for a learning object more or less mapped out and knew in principle that technology was capable of supporting a solution that would allow students to build and reinforce knowledge through repeated interaction. What she lacked was specific knowledge of how to implement that solution.

The solution to the AIS problem emerged during a meeting with a highly experienced chemistry lecturer who had developed the BestChoice interactive tutorial system (http://130.216.56.150/). This teacher had considerable experience of developing computer-based learning activities gained through over 10 years of practice. BestChoice is a database-driven system in which different types of feedback are a key feature:

- Users receive immediate feedback commensurate with their responses to a range of tutorial questions.

- Users can easily give context sensitive feedback to assist with system improvements.

- System log data provides aggregate and individual performance data to inform revision and further development.

The chemistry teacher has remained at the leading edge of science education methods and sought solutions to numerous learner challenges through additions and extensions to the BestChoice system. Many refinements were based on suggestions from student users. Thus the database structure not only supports student engagement with the content, but is also a repository of data on user interactions and comments.

Some readers might argue that BestChoice is not a learning object because it is too large and complex to fit standard definitions, or because it does not include metadata describing the content or pedagogical contexts for use. However, the developer has 
always considered it to be a learning object, it fits the definition used in this paper and that definition is derived from other published sources. Regardless of its acceptance, successful articulation of the concept of reusability is demonstrated. The process now described has already proved to be sustainable and successful in ways that other reusability initiatives have not. The difference lies in the participative approach to repurposing rather than simple reuse of learning objects.

\section{Repurposing a learning object}

The collaboration began following a presentation by the BestChoice designer. She confirmed that the conceptual solution developed for the AIS students fell within the capability of the existing system. For the activities, five sets of exercises designed to support incremental development of diagramming skills translated into 25 interactive web pages with short narratives accompanying graphical representations of the tasks for students to complete. Constructive feedback was written for both correct and incorrect responses, as a 'scaffolded' approach is preferred. Content submitted by the AIS teacher was translated into web format by the BestChoice developer, who judged that the system's resident web editor would be too complicated for the novice AIS user to work with independently. Ongoing dialogue over a period of four weeks supported development and later refinement of the online tasks. Specific design features were negotiated, with the tasks easily structured to fit into the extensive range of question types provided by BestChoice. No compromise of planned activity design proved necessary. A 'trial and error' (heuristic) approach was the common language of negotiation and both parties developed deeper understanding of the system. The AIS teacher learned about functionality, structure and capability. The BestChoice developer learned how to repurpose the structure for use in different subject areas and learning designs. Perhaps the hardest part for the AIS teacher was developing deep conceptual understanding of the database structure behind the system; that is, of the technology that supports the pedagogy. However, the ability to derive that understanding from direct experience of developing a version of the system in the meaningful context of her own teaching practice did much to facilitate the learning process. After one participative venture, she felt confident enough to work independently with the BestChoice web editor and development tools, and competent to do so with her newly developed understanding of the technology. The BestChoice developer's teaching expertise allowed her to gauge how her collaborator's understanding progressed, how best to support this and take it to the next stage. It is probable that the developer's own incrementally acquired understanding informed her actions in this respect.

To summarize the outcomes of the collaboration, instructional design, technical expertise and intimate knowledge of student behaviour with interactive systems contributed by the BestChoice developer complemented the pedagogical content knowledge of the subject expert to successfully repurpose the learning object for use in another disciplinary context. The structural elements used for chemistry applied equally well to content and learning activities for AIS. The setting in which this 
initiative took place is typical of the contemporary university context; that is, characterized by increasing class size and diversity, financial constraints and limited resources for teaching. The methodological shift towards focus on learning rather than teaching makes the case for repurposing learning objects (or systems) in this manner compelling.

\section{Discussion}

The participative design approach described in this paper addresses critical issues of deep conceptual understanding of the role and scope, as well as acceptance and ownership, of reusable learning objects. The long-term goal of educational culture change, which is the final stage of the technology acceptance model, begins to be addressed with the repurposing model. The prospect of achieving similar degrees of success with teacher professional development and sustainable impact is less certain with other initiatives such as learning object repositories, dissemination or generic staff development programmes. Such initiatives generally do increase knowledge and promote positive moves along the acceptance continuum. However, the overall impact is less significant than when the repurposing approach is applied. Similarly, the removal of technical barriers, establishment of interoperability standards, metadata and cataloguing systems are positive dimensions of accessibility. However, they are not sufficient in themselves to drive the academic culture change that is needed to promote the emergent learner-centred education model that uses learning objects as a core resource. Nor are they likely to do so on the same scale as the 'participative design in repurposing' model. For this shift to occur, the conceptual models of curriculum developers and teachers need to embrace the concept of reusability in a meaningful way. This implies a sense of ownership through participation in design and development as well as the ability to drive innovation to new levels. Like any other conceptual development, there is no easy route to achievement of this high level of proficiency, as the case study illustrates.

The AIS lecturer began as a novice with the concepts of learning object design and reuse. After four weeks of intensive collaborative work to repurpose a learning object structure for use within her own subject area, she was capable of producing fully interactive online activities independently of her mentor. She had also analyzed student learning and reviewed her teaching strategies in a detailed way. This in-depth analysis resulted from the need to express the principles underlying her teaching practice and to translate them into online learning activities using digital media format. She is now competent to apply this enhanced conceptual understanding to the development of learning objects in other topic areas. She is also in a position to share the AIS learning object and her experience of participative development with colleagues in her department, and perhaps to drive future developments of similar nature. This level of ability could not be achieved by other means, particularly within the period of 15 working days of the repurposing initiative.

The learning object structure originally designed for interactive chemistry tutorials has proved to be reusable in different subjects. The development of BestChoice 
involved considerable effort, resource commitment and a steep learning curve. However, the resultant benefits to students, staff and the e-learning community are significant. In future, these benefits will extend to the international AIS community, as the diagramming object will be added to a shared resource collection that is currently under development. An existing AIS community of practice provides the means for dissemination. It is the nature of communities of practice to self-generate and perpetuate. Perhaps the lesson here is that the learning object economy will develop through the same means rather than being driven by the architects of the original technology.

To teachers who fear an 'identity crisis' caused by e-learning 'commodification', the repurposing concept may bring a sense of relief. Professional expertise is a critical part of the innovation process and different teaching styles an integral part of the learning environments that reusable learning objects can be designed for and integrated into. As to what the 'dominant design' for learning objects or courses that incorporate them will be, if such a design emerges, as the literature on innovation cycles suggests it must, the teachers involved in repurposing initiatives are best placed to contribute to its definition.

\section{References}

Barnett, J. \& Hodson, D. (2001) Pedagogical content knowledge: toward a fuller understanding of what good science teachers know, Science Education, 85(4), 426-453.

Boyle, T. \& Cook, J. (2001) Towards a pedagogically sound basis for learning object portability and reuse, in: M. Keppel et al. (Eds) Meeting at the crossroads. Proceedings of the 18th Annual Conference of the Australian Society for Computers in Learning in Tertiary Education (Melbourne, Biomedical Education Unit, University of Melbourne), 101-109.

Burbules, N. \& Callister, T. (2000) Watch IT: the risks and promises of information technologies for education (Boulder, CO, Westview Press).

Campbell, L. (2003) Engaging with the learning object economy, in: A. Littlejohn (Ed.) Reusing online resources: a sustainable approach to e-learning (London \& Sterling, VA, Kogan Page), $35-45$.

Darby, J. (1992a) Computers in teaching and learning in UK higher education, Computers in Education, 19(1-2), 1-8.

Darby, J. (1992b) The future of computers in teaching and learning, Computers in Education, 19(1-2), 193-197.

Downes, S. (2001) Learning objects: resources for distance education worldwide, International Review of Research in Open and Distance Learning, 2(1). Available online at: http:// www.irrodl.org/content/v2.1/downes.html (accessed 15 July 2003).

Fullan, M. (2001) The new meaning of educational change (New York, Teachers College Press).

Gardner, N. \& Darby, J. (1990) Using computers in university teaching: a perspective on key issues, Computers and Education, 15(1-3), 27-32.

Hammond, N., Gardner, N., et al. (1992) Blocks to the effective use of information technology in higher education, Computers and Education, 18(1-3), 155-162.

Hodas, S. (1996) Technology refusal and the organizational culture of schools, in: R. Kling (Ed.) Computerisation and controversy: value conflicts and social choices (San Diego, Academic Press).

Hurley, J. (1992) Towards an organizational psychology model for the acceptance and utilisation of new technology in organizations, The Irish fournal of Psychology, 13(1), 17-31. 
Littlejohn, A. (2003) Reusing online resources: a sustainable approach to e-learning, Open and flexible learning series (London \& Sterling, VA, Kogan Page).

Nichols, M. (2002) Education and learning objects: a primer, UCOL. Available online at: http:// www.cta.ac.nz/papers/LearningObjectsCPIT.doc (accessed 23 October 2003).

Priest, A. \& Quaife-Ryan, M. (2004) Re-enchanting education: the recovery of teaching as a sacred activity, Lifelong Learning Conference Proceedings (Rockhampton, Central Queensland University), 300-306.

Taylor, P. (1998) Institutional change in uncertain times: lone ranging is not enough, Studies in Higher Education, 23(3), 269-279.

Taylor, P. \& Richardson, A. (2001) Validating scholarship in university teaching: constructing a national scheme for external peer review of ICT Based teaching and learning resources, Report 03/ 01, Evaluations and Investigations Programme, Higher Education Division, Department of Education Training and Youth Affairs. Available online at: http://www.deetya.gov.au/divisions/hed/operations/eippubs.htm (accessed 22 June 2002).

Wiley, D. (2000) Connecting learning objects to instructional design theory: a definition, a metaphor and taxonomy, in: D. Wiley (Ed.) The instructional use of learning objects. Available online at: http://reusability.org/read/chapters/wiley.doc (accessed 14 April 2003).

Wilson, S. (2001) The next big thing? Three architectural frameworks for learning technologies, The Centre for Educational Technology Interoperability Standards. Available online at: http:// www.cetis.ac.uk/content/20010828163808/viewArticle (accessed 2 May 2003).

Zemsky, R. \& Massy, W. (2004) Thwarted innovation: what happened to e-learning and why?, Final report of the Weatherstation Project, The Learning Alliance, University of Pennsylvania. Available online at: http://www.irhe.upenn.edu/WeatherStation.html (accessed 4 March 2005). 